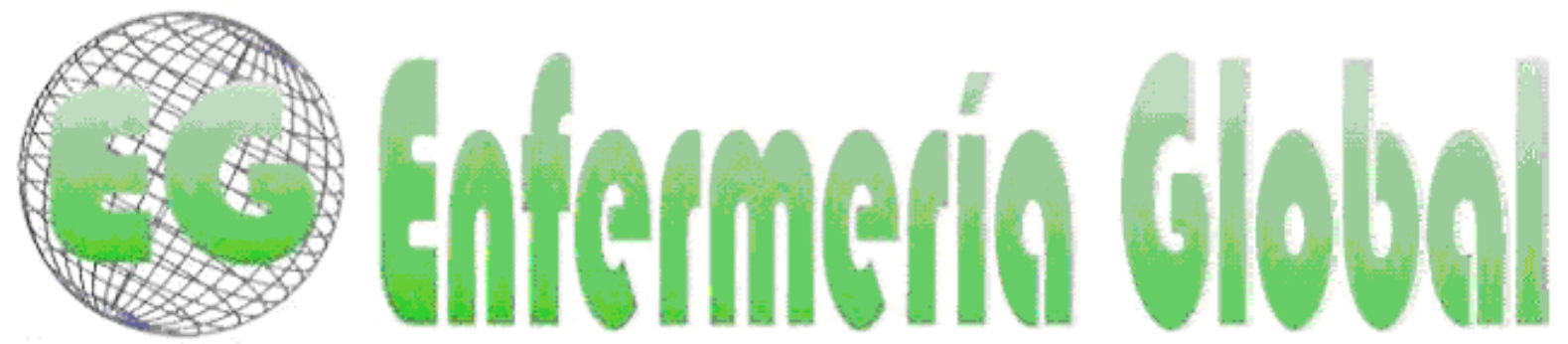

\title{
Implementación de subdirecciones de gestión del cuidado en hospitales autogestionados, avances y desafios para enfermería
} Care management subdivision implementation in self managed hospitals, improvements and challenges for nursing

\author{
*Mosqueda Díaz, A., **Stiepovich Bertoni, J. \\ *Enfermera - Matrona, Docente Escuela de Enfermería Universidad de Valparaíso. E-mail: \\ amosqueda@udec.cl **Doctora en Enfermería, Docente Programa de Doctorado Universidad de \\ Concepción. Chile.
}

Palabras clave: Enfermería; Gestión en salud; Garantía de la Calidad de Atención de Salud

Keywords: Nursing; Health Management; Quality Assurance of Health Care

\section{RESUMEN}

En Chile se ha implementado una reforma sanitaria, entre las modificaciones plantea la creación de Establecimientos Autogestionados en Red (hospitales de alta complejidad con autonomía en la gestión), entre los requisitos que deben cumplir para logar esta calidad es acreditarse como prestadores de salud, lo que exige implementar "Subdirecciones de Gestión del Cuidado".

El presente artículo tiene por objetivo examinar el grado de avance en la implementación de la reforma sanitaria y el rol que a enfermería le compete en ella, se analizan críticamente los desafíos que se presentan a nivel profesional, y se reflexiona respecto a los alcances para la disciplina y la formación de enfermería.

Como metodología se realizó una revisión bibliográfica en las bases de datos PubMed, Scielo, Redalyc y Bireme.

El resultado del análisis de los documentos generó cuatro áreas temáticas: Reforma Sanitaria en Chile, Implementación de los Establecimientos Autogestionados en Red, Aseguramiento de la Calidad, y Gestión del Cuidado y formación profesional.

Entre las conclusiones destaca que la revisión bibliográfica no arrojó evidencias para determinar si la implementación de las Subdirecciones de Gestión del Cuidado han generado algún impacto en la calidad de los cuidados otorgados a los usuarios, lo que podría deberse al tiempo de su implementación, sin embargo se espera que los procesos de acreditación aporten experiencias publicables al respecto. 


\section{ABSTRACT}

In Chile a sanitary reform has been launched, among other modifications, it shows the option to create Self Managed Network Institutions (high complexity hospitals with autonomous management). To get this Grade, there are some requirements to comply with, between them; they must be accredited as Health Providers, which demands an implementation of "Care Management Subdivisions".

This Paper aims to assess the implementation process status of the sanitary reform and the Nursing role in it. It critically examines the challenges that are presented at a professional level and it ponders over the scope towards the discipline and nursing training as well.

As methodology, bibliographical research was carried out on data based on PubMed, Scielo, Redalyc and Bireme. The outcome pointed out four thematic areas: Sanitary Reform in Chile, Implementation of Self Managed Network Institutions, Quality Assurance, Care Management and Professional Training.

Among the conclusions, it is highlighted that the bibliographic research could not give any evidence to determine whether the implementation of Self Managed Network Institutions have made any change in the impact on quality care provided to users, which could be caused by implementation time, nevertheless it is expected that accreditation processes will bring up related experiences that can be published.

\section{INTRODUCCIÓN}

En las últimas décadas, Chile ha mejorado los indicadores de salud, acercándose a los de países desarrollados. Para lograrlo, sin duda, ha influido la cobertura de los servicios básicos y el nivel educacional de la población, esto ha significado un cambio respecto a los problemas de salud relevantes, hacia aquellos asociados con la avanzada transición demográfica y las variaciones en el perfil epidemiológico de la población ${ }^{(1)}$.

Para enfrentar esta nueva situación, se implementó una reforma sanitaria que incluye profundas modificaciones al sistema de salud, tanto en su estructura como en su funcionamiento.

Este documento tiene por objeto revisar los cambios relacionados con la instauración de los Establecimientos Autogestionados en Red (EAR) y las Unidades de Gestión del Cuidado, como parte de la reforma; a través de una revisión bibliográfica, se analizaron los antecedentes que sustentaron su implementación, los avances alcanzados y los desafíos que plantea para Enfermería como profesión.

Por otra parte, la mencionada reforma sanitaria también explicita un sistema de aseguramiento de la calidad, a través de procesos de acreditación, donde los hospitales deben cumplir con estándares mínimos para garantizar la seguridad de las prestaciones que brindan. El concepto de "calidad" utilizado es el planteado por Donabedian ${ }^{(2)}$, que involucra componentes de estructura, proceso y resultado; al respecto se revisan las implicancias para Enfermería y los aspectos disciplinarios y éticos que deben ser considerados.

Finalmente, se realiza una propuesta de abordaje curricular para las carreras de Enfermería, respecto a estos temas, con el fin de aportar al mejoramiento de la formación de los nuevos profesionales en este nuevo contexto de salud. 


\section{METODOLOGÍA}

Según los objetivos propuestos, se realizó una revisión bibliográfica descriptiva y exploratoria para conocer el estado del arte respecto a la implementación de las subdirecciones de gestión del cuidado en hospitales autogestionados, y los aportes por parte del profesional de enfermería al respecto.

Para el levantamiento bibliográfico se utilizaron documentos legales, publicaciones en medios de difusión local y artículos incorporados en las bases de datos PubMed, Scielo, Redalyc y Bireme, publicados entre el año 2000 y 2011, la búsqueda fue realizada durante los meses de abril y mayo del año 2011 y se consideraron como descriptores iniciales: gestión del cuidado, hospital autogestionado y calidad acreditación hospitalaria.

Posteriormente se realizó la lectura y análisis crítico de los documentos, lo que permitió sistematizar la información en cuatro áreas temáticas principales: Reforma Sanitaria en Chile, Implementación de los EAR, Aseguramiento de la Calidad, y Gestión del Cuidado y formación profesional. Esto posibilitó la construcción de los resultados así como también su discusión.

\section{RESULTADOS Y DISCUSIÓN.}

\section{Reforma Sanitaria en Chile.}

Como parte de una política para enfrentar la situación de salud en la década 2000 2010, se generó un equipo interdisciplinario que realizó un diagnóstico de salud y que estableció cuatro objetivos sanitarios, que determinaron las prioridades para el sector $^{(3)}$ :

1. Mejorar los logros sanitarios alcanzados.

2. Enfrentar los desafíos derivados del envejecimiento y los cambios de la sociedad.

3. Disminuir las desigualdades.

4. Prestar servicios acordes a las expectativas de la población.

Para cumplir estos objetivos, se creó un comité interministerial que trabajó entre los años 2000 y 2005, cuya función fue generar los proyectos de ley necesarios para dar sustento a la reforma de salud propuesta ${ }^{(4)}$, el trabajo de este comité concluyó con la aprobación de cinco leyes que regulan: el financiamiento, el funcionamiento las instituciones previsionales de salud, un plan de salud obligatorio para enfermedades de alto impacto sanitario y social, y la gestión de la autoridad sanitaria.

Es así como, en el año 2004, fue aprobada la Ley № 19.937 conocida como "Ley de Autoridad Sanitaria", que tuvo por finalidad "...establecer una nueva concepción de la autoridad sanitaria, distintas modalidades de gestión y fortalecer la participación ciudadana" ${ }^{(5)}$. Entre las principales modificaciones generadas se encuentran:

- La creación de dos Subsecretarias: Una de Redes Asistenciales encargada de la prestación de servicios de salud y otra de Salud Pública a cargo de la promoción, vigilancia, prevención y control de enfermedades.

- La creación de la Superintendencia de Salud.

- El funcionamiento de los organismos de salud en sistema de red.A nivel regional se redistribuyeron funciones, de manera que las Secretarias Regionales 
Ministeriales de Salud (SEREMI) asumían labores de Salud Pública y los Servicios de Salud se redefinían como "Gestores de Redes".

- Y la creación de la figura de los "Establecimiento Autogestionados en Red" (EAR).

En términos generales, las modificaciones planteadas se direccionan hacia dos puntos críticos: la gestión de los servicios sanitarios y el sistema de atención ${ }^{(6)}$; esto con el propósito de "Mejorar la salud de la población, prolongando la vida y los años de vida libre de enfermedad $y$, reducir las desigualdades en salud, mejorando la salud de los grupos más desfavorecidos de la sociedad" (3). Al respecto, algunos autores ${ }^{(4)}$ han señalado en su favor que se trata de una "reforma con enfoque de derecho", y otros la critican indicando que es un proceso más orientado a la reingeniería que hacia el fortalecimiento de la autonomía en la gestión ${ }^{(7)}$.

Es necesario señalar que el sistema de salud operante en Chile hasta los años noventa, requería modificaciones importantes y por lo investigado hasta ahora, la génesis de este proyecto se sustentó en mejorar los principales problemas vivenciados: débil articulación de los actores en salud, desigualdades de acceso, centralización en la toma de decisiones y en la distribución de recursos, entre otras. Sin embargo, su implementación no ha estado exenta de dificultades.

\section{Implementación de los Establecimientos Autogestionados en Red.}

Para adquirir la calidad de "Establecimientos Autogestionados en Red (EAR)", más conocido como "hospitales autogestionados", se requiere del reconocimiento por el correspondiente Servicio de Salud como establecimientos de mayor complejidad técnica, esto significa que cuentan con a lo menos una especialidad básica y varias subespecialidades $\mathrm{y}$, además deben someterse a un proceso de evaluación de cumplimiento de requisitos, con todo esto el Ministerio de Salud y Ministerio de Hacienda elaboran una resolución conjunta que les otorga la mencionada calidad ${ }^{(5,8)}$.

Los requisitos que la Ley establece como estándares mínimos para conseguir la calidad de EAR, están relacionados con la organización, gestión en redes, gestión de recursos humanos, gestión financiera, gestión de calidad e implementación de mecanismos de participación ${ }^{(5,8)}$. La figura 1 presenta un detalle de los aspectos considerados en cada uno de estos puntos. 


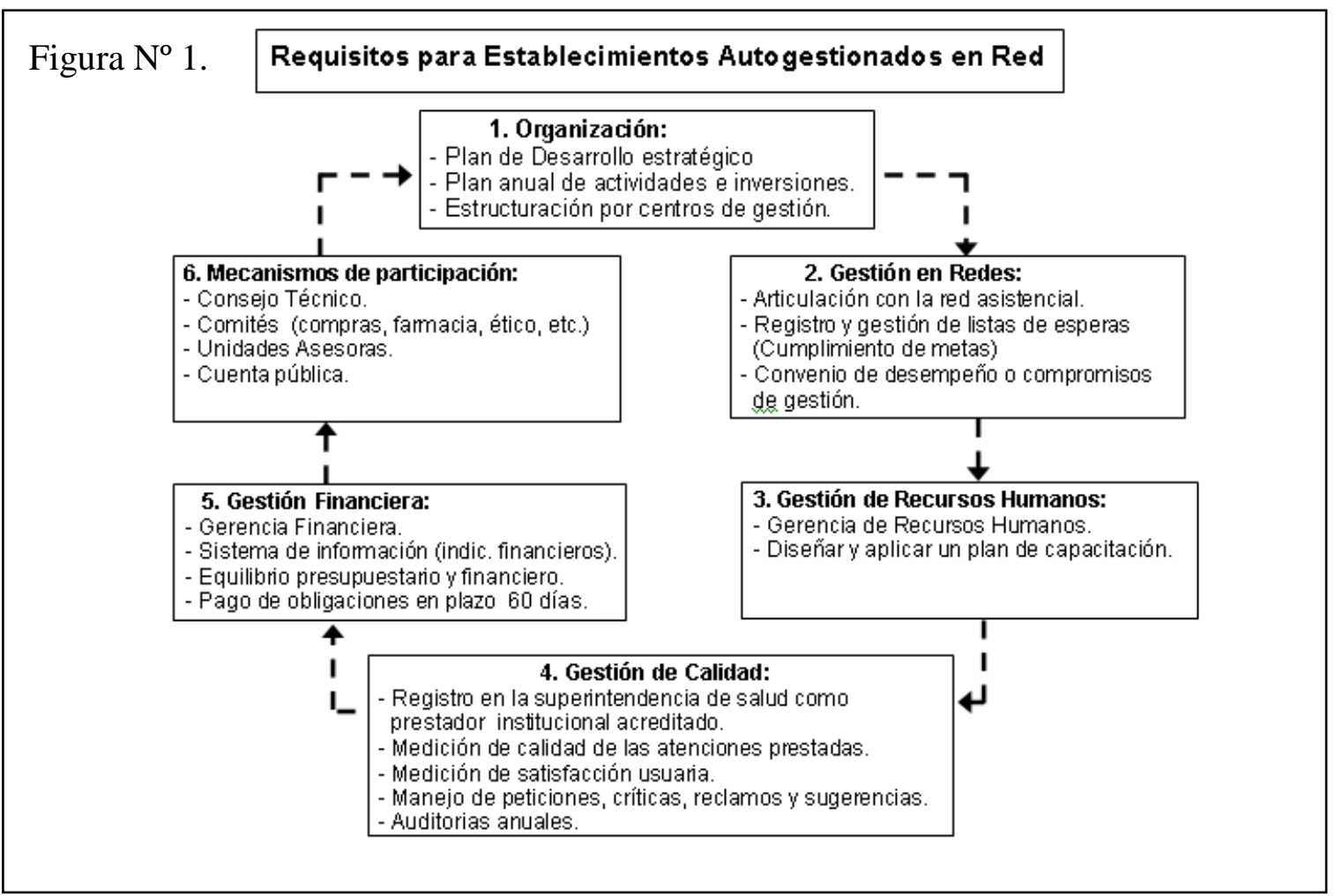

Fuente: Adaptación Mosqueda y Stiepovich 2011, de lo indicado en la Ley 19.937 Art. 25G.

Según se desprende de los requisitos presentados, los EAR son concebidos como órganos con atribuciones desconcentradas del correspondiente Servicio de Salud, son vistos bajo un modelo gerencial de empresa sustentable, donde se les exige la aplicación de herramientas tecnológicas, clínicas y de recursos (financieros, humanos y materiales), para el cumplimiento de las metas y objetivos sanitarios ${ }^{(5,8)}$.

Se esperaba que el 1 de enero del año 2009 todos los hospitales contaran con la calidad de EAR, sin embargo, para fines del 2008 sólo 11 (de 59) habían conseguido el nombramiento. A la luz de estos antecedentes, el Ministerio de Salud planteó al parlamento que "Ios hospitales no se encontraban preparados para enfrentar esta nueva condición" ${ }^{(1,4)}$, por lo que se acordó aplazar el proceso por un año y, conjuntamente el desarrollo de programas de capacitación y acompañamiento que les permitiera a los hospitales lograr los referidos nombramientos. Una vez cumplido el nuevo plazo y a pesar de no haber cumplido con el $100 \%$ de los requisitos exigidos, los hospitales de mayor complejidad serían nombrados por Ley como EAR ${ }^{(10,11)}$.

Se han señalado diversas causas para explicar las dificultades que han tenido los hospitales para lograr la calidad de EAR, entre ellas destacan: que los hospitales no han alcanzado los estándares de calidad establecidos, ausencia de liderazgo para generar los cambios organizacionales, que los diferentes actores no han asumido sus nuevos roles ${ }^{(1)}$, incapacidad de planificación para satisfacer los requerimientos de los gestores y del sistema, falta de políticas de recursos humanos acorde a nuevos requerimientos ${ }^{(7)}$ y la falta de sistemas de control de gestión interno y externo ${ }^{(10)}$. Al analizar las razones planteadas se observa que los autores, en general, enfatizan las responsabilidades hacia unos $u$ otros actores de la reforma; sin embargo, se debe considerar que los profundos cambios que ésta involucra, la intrincada suma de factores que deben articularse, las nuevas responsabilidades que deben asumirse y las exigentes metas establecidas, hacen que cada uno de los integrantes deba estar 
preparado individual y colectivamente y, en este sentido la preparación necesaria y el tiempo requerido para su implementación pudieron estar subestimados.

\section{Aseguramiento de la Calidad de los Establecimientos Autogestionados en Red.}

\subsection{Procesos de Acreditación}

La Ley de "Autoridad Sanitaria" (5) indica que los establecimientos de salud deben someterse a procesos de acreditación, cuya función es "evaluar el cumplimiento de los estándares fijados, con el objeto de velar porque las prestaciones que se otorgan revistan la calidad necesaria para resguardar la seguridad de sus usuarios" (11), esta norma general está concebida tanto para organizaciones públicas como privadas.

A pesar de lo planteado por la ley, el requisito con menor cobertura de cumplimiento de los EAR es: el registro en la Superintendencia de Salud como prestador acreditado. Hasta abril del año 2011, existen sólo 7 prestadores de atención cerrada (hospitales) acreditados, y de ellos sólo 1 corresponde a un EAR ${ }^{(12)}$. Los hospitales no lograron cumplir los requisitos en los nuevos plazos establecidos por el parlamento para ser EAR y finalmente les fue designada esta calidad por Ley.

Esta situación ha sido considerada de tal importancia, que fue definida como el Compromiso de Gestión No 18 por el actual Gobierno. Para enfrentarlo "se ha diseñado un plan con iniciativas de capacitación, apoyo tanto a nivel local (hospitales y servicios de salud) como central, elaboración de guías metodológicas y planillas que permitan contar con información actualizada de las mediciones, cumplimientos de los estándares generales... y estado de avance..." (13), con esto se persigue que a fines del año 2011 los EAR estén en condiciones de iniciar el proceso formal de acreditación.

Los procesos de acreditación no tienen un modelo único, autores señalan que deben adaptarse a la propuesta política que lo sustenta ${ }^{(14)}$; en el caso chileno, se basa en la experiencia norteamericana de la agencia "Joint Comission on acreditation of health care organizations" (15), la que utiliza el marco teórico conceptual de Donabedian (2) (citado por Penha), donde los procesos evaluativos de los servicios de salud deben contemplar un análisis en relación con la estructura: recursos físicos, humanos y financiero, de proceso: actividades desarrolladas entre el personal de salud y el usuario y, de resultado: cambios producidos en el usuario por el cuidado recibido y, en relación a ellos establecer estándares consensuados y uniformes que marcan expectativas alcanzables para las instituciones ${ }^{(2,16)}$.

El proceso se inicia con una autoevaluación realizada por los mismos prestadores de servicios, aquí se documentan y evalúan las actividades de mejora continua de la calidad de la atención implementada, esta autoevaluación puede ser aplicada al establecimiento en su funcionamiento general o a prestaciones determinadas; luego continúa con una evaluación por parte de una entidad acreditadora, quien contrasta las condiciones del prestador con los estándares establecidos, con la finalidad de determinar su cumplimiento. Como resultado de este proceso, el prestador puede ser acreditado, no acreditado o acreditado con observaciones. En este último caso debe presentar un programa de mejoramiento que será reevaluado en el plazo de seis meses y con ello se determinará si acredita o no, la no acreditación significa la pérdida de recursos económicos para el funcionamiento del EAR ${ }^{(11)}$. 
Las exigencias para obtener la acreditación van aumentando progresivamente, es así que, para acreditar por primera vez se requiere del cumplimiento del $50 \%$ de las características aplicadas, en la segunda oportunidad el $70 \%$ y, a partir de la tercera vez el cumplimiento debe alcanzar el 95\%. La acreditación debe realizarse cada tres años ${ }^{(17)}$.

Los procesos de acreditación exigen de los establecimientos una profunda mirada al trabajo realizado, como todo proceso evaluativo genera situaciones de incertidumbre que pueden tensionar el clima organizacional, sin embargo, es una herramienta útil para implementar el concepto de mejora continua y aún más, que ello se refleje en la calidad de la atención otorgada a los usuarios.

Son ocho los ámbitos evaluados en el proceso de acreditación institucional, a saber $^{(17)}$ : Respeto a la dignidad del paciente, Gestión de la calidad, Gestión clínica, Acceso, oportunidad y continuidad de la atención, Competencias del recurso humano, Registros, Seguridad del equipamiento y Servicios de Apoyo (laboratorio clínico, farmacia, esterilización, transporte de pacientes, diálisis, radioterapia, anatomía patológica, quimioterapia, imaginología, kinesioterapia y rehabilitación kinésica, medicina transfusional, atención de donantes y centro de sangre).

\subsection{Desafíos planteados por la Acreditación a la Profesión de Enfermería.}

Con la incorporación del artículo 113 al "Código Sanitario" en el año 1997, el rol asignado legalmente en Chile a la enfermera/o identifica tres grandes aspectos ${ }^{(18)}$ : La gestión de cuidado, la ejecución de acciones derivadas del diagnóstico y tratamiento médico y la mejor administración de recursos de asistencia para el paciente. Todos intervienen en la calidad de la atención otorgada a los usuarios, no obstante, nos centraremos en analizar el primero de ellos, por su gravitación en la implementación de los EAR.

La Ley de "Autoridad Sanitaria" establece como requisito mínimo para la creación y funciones de los EAR " ...que deberán estar referidos, al menos, al cumplimiento de metas y objetivos sanitarios, a gestión financiera, gestión de personal, gestión del cuidado e indicadores y estándares fijados en convenios y normas" ${ }^{(5)}$. Esto para Enfermería, en la práctica se tradujo en la generación de un cargo de alta responsabilidad directiva con la creación de las "Subdirecciones de Gestión del Cuidado" y modificaciones de las líneas de dependencia del personal de enfermería directamente de esta subdirección y no de las jefaturas médicas como era anteriormente ${ }^{(19,20)}$. Su implementación ha requerido de una fundamentada defensa por parte de las/os enfermeras/os, ya que ha sido vista como una amenaza bajo la mirada del paradigma biomédico, o ha querido ser asumido por otros profesionales aludiendo que también desempeñan actividades de "gestión del cuidado" (21), pese a ello su implementación ha continuado en forma progresiva a nivel nacional.

En el año 2007, se aprobó la Norma General Administrativa No 19: "Gestión del cuidado de enfermería para la atención cerrada", que es entendida como: "el ejercicio profesional de la enfermera sustentada en su disciplina, la ciencia del cuidar, se define como la aplicación de un juicio profesional en la planificación, organización, motivación y control de la provisión de cuidados oportunos, seguros, integrales, que aseguren la continuidad de la atención y se sustenten en las políticas y lineamientos estratégicos de la institución" ${ }^{(19)}$. Esto implica, que Enfermería tiene la histórica 
oportunidad de demostrar que cuenta con las competencias profesionales y disciplinares para asumir las tareas que legalmente se le han conferido.

Las atribuciones otorgadas por el Ministerio de Salud a la enfermera/o como encargada de la gestión del cuidado son: "organizar, supervisar, evaluar y promover el mejoramiento de la calidad de los cuidados de enfermería" ( ${ }^{19)}$; para desarrollar estas funciones se han reconocido habilidades claves, tales como: liderazgo, comunicación efectiva, capacidad de trabajo en equipo y de toma de decisiones, a través de las cuales, el profesional de enfermería motiva a su equipo para entregar cuidados oportunos, continuos y de calidad ${ }^{(22-24)}$. De esta forma, los procesos de acreditación pueden convertirse en el instrumento a través del cual se muestre objetivamente el quehacer de enfermería como gestora de cuidados.

A modo de ejemplo se presentan algunos de los ámbitos considerados en la acreditación ${ }^{(17)}$ y los aportes que puede realizar la/el enfermera/o:

\section{0 Ámbito: Respeto a la Dignidad del Paciente (DP).}

DP-1.3: "Se evalúa el efectivo respeto de los derechos de los pacientes, incluyendo mediciones de la percepción de los pacientes y demás usuarios".

El profesional de Enfermería, desde su conocimiento disciplinar, podría aportar con la aplicación de modelos y teorías que sustenten esta nueva mirada a los usuarios y liderar el grupo encargado de la actividad.

DP- 5.1: "El prestador institucional dispone de, o tiene acceso a, un comité de ética donde se resuelven las material de orden ético que se suscitan como consecuencia de la labor asistencial."

Los enfermeros/as están llamados a adquirir competencias específicas en el área de la ética y la bioética, para ser parte activa de los comités de ética y aportar desde la mirada del cuidado profesional.

\section{2ํ Ámbito: Gestión de la Calidad (CAL).}

CAL-1.2: "Existe un responsable de coordinar las actividades de mejoría continua de la calidad en las unidades, y se definen metas de calidad anuales en dichas unidades."

Esta responsabilidad puede y debería ser asumida por un enfermero/a, por lo que es necesario profundizar los conocimientos en gestión y aplicación de metodologías de mejoramiento continuo de la calidad.

\section{3o Ámbito: Gestión Clínica (GCL).}

GCL- 1.2: "Se aplica un programa de evaluación y mejoría de las prácticas clínicas: Atención de Enfermería."

Es en este ámbito donde las capacidades del profesional de enfermería pueden tener su máximo desarrollo como gestor del cuidado, existen estudios que documentan la influencia positiva sobre la calidad de la atención cuando los cuidados son proporcionados o gestionados por enfermeras/os ${ }^{(25,26)}$. Actualmente el desafío es 
mostrar resultados en este sentido, a través de la generación de programas de mejoramiento continuo participativos, motivación al equipo de enfermería en la implementación de soluciones y evaluación de su impacto a través de instrumentos validados. En la medida en que se muestren objetivamente los logros alcanzados, podría ser un referente para otras áreas donde el profesional de enfermería puede aportar su experiencia a través de asesorías.

En resumen, en todos los ámbitos del proceso de acreditación la enfermera/o puede realizar aportes significativos, ahora solo depende de su capacidad para adaptarse y asumir como protagonista este desafío.

\subsection{Evaluación de la Calidad del Cuidado de Enfermería.}

En las últimas décadas, numerosos países han implementado reformas sanitarias, éstas han derivado en un creciente interés por objetivar la calidad de la atención de salud, en general, y de los cuidados de enfermería, en particular. Esto hace posible encontrar múltiples publicaciones con investigaciones relacionadas al tema de calidad en salud, desde distintas perspectivas: evaluación procedimental ${ }^{(16)}$, percepción de calidad por el usuario ${ }^{(27-30)}$, satisfacción del usuario interno ${ }^{(31)}$, entre otros. Se escogieron algunos artículos con la finalidad de analizar los aspectos convergentes y divergentes que poseen al abordar la temática.

\section{Como aspectos comunes se encontró que:}

En general utilizan el modelo de análisis de calidad propuesto por Donabedian ${ }^{(2)}$, el cual no siempre es reconocido explícitamente, sin embargo, los instrumentos propuestos contemplan aspectos de estructura, de proceso y de resultado ${ }^{(16,27-31) \text {. }}$

- Varios concuerdan en que la calidad de la atención otorgada depende de: las expectativas que tenga el usuario de ella y, de cómo percibió la satisfacción de sus necesidades durante su interacción con el medio asistencial, por ello cobra relevancia las mediciones de percepción y justifican su realización ${ }^{(27-31)}$.

La diferencia más significativa radica en que los instrumentos utilizados varían en su conformación, integrando distintas dimensiones y número de ítems, lo que dificulta realizar análisis comparativos.

Se debe puntualizar que en la revisión bibliográfica realizada, no se encontraron artículos elaborados por profesionales de enfermería chilenos que puedan mostrar los aportes de la implementación de las Unidades o Subdirecciones de Gestión del Cuidado en los EAR, o cómo éstos han impactado en la calidad de los cuidados otorgados a los usuarios, sin duda el corto tiempo trascurrido desde su instauración y las dificultades en su implementación han influido en ello. Sin embargo, se espera que las experiencias y resultados obtenidos en los procesos de acreditación que se desarrollan durante el presente año, se constituyan en información relevante para futuras publicaciones.

\subsection{Gestión del Cuidado: Un análisis desde lo Ético y lo Disciplinar.}

Ante la siguiente interrogante, ¿por qué Enfermería debe preocuparse de la calidad de los cuidados otorgados?, algunos indican que es una responsabilidad ética ${ }^{(28,31)}$, es una responsabilidad que implica no solo una forma de hacer sino también de ser, 
"los cuidado de enfermería no se limitan a exigir una competencia técnica sino que deben estar atravesados por un sentido humano" (32).

Al respecto, la "Gestión del Cuidado" es una oportunidad para demostrar que Enfermería posee las competencias científicas, técnicas y humanas para asegurar la calidad de la atención que se brinda a los usuarios, sin embargo, algunos autores indican que "las enfermeras(os) han centrado su quehacer en lo administrativo, dejando en segundo plano el deber de velar por la persona" ${ }^{(33)}$ y, desde la ética se ha cuestionado la calidad de los cuidados cuando estos son desarrollado en contextos de "deshumanización" (32). Todo esto hace necesario volver a la esencia de Enfermería y recordar que: siendo la gestión un ámbito de desempeño declarada por la profesión, esta se justifica solo en el hecho de ser un aporte para mejorar los cuidados.

Lo planteado no es sólo un fenómeno local, ha sido analizado por Jean Watson, una de las grandes teoristas contemporáneas de Enfermería, autora de la "Filosofía y ciencia del Cuidado", quien señala que "Ante el riesgo de deshumanización en el cuidado de las personas, a causa de la gran reestructuración administrativa de la mayoría de los sistemas de cuidado de salud en el mundo, se hace necesario el rescate del aspecto humano, espiritual y transpersonal, en la práctica clínica, administrativa, educativa y de investigación por parte de los profesionales en el campo de la enfermería" (34).

Por lo tanto, la calidad en el cuidado profesional de enfermería debe ser una premisa sustentada en sus bases disciplinares y éticas, donde el cuidado sea la razón de ser del quehacer de enfermería y la gestión una herramienta centrada en mejorarlo, es decir, "El cuidado debe movilizar las actividades de gestión implementadas".

\section{Gestión del Cuidado y la Formación del Profesional de Enfermería.}

En Chile existen 13 carreras de Enfermería con acreditación vigente, impartidas por universidades públicas y privadas.

Se revisó la información que entregan a través de las páginas web institucionales encontrándose que: sólo 5 de ellas declaran explícitamente en el perfil de egreso competencias en "Gestión del Cuidado", y 6 incorporan asignaturas con nombres que incluyen términos relacionados con "Gestión del Cuidado o Gestión en Enfermería". Sin duda, sería necesario realizar una investigación que profundizara los aspectos mencionados e incluyera las temáticas abordadas.

Por otro lado, en la actualidad se vive un fuerte movimiento de innovación curricular a nivel nacional, sería conveniente emplear esta instancia para que las carreras explicitaran la "Gestión del Cuidado" como eje del perfil de egreso, e incluir en el plan curricular y los programas que lo componen su análisis y aplicación. Sería de gran valor que las carreras incorporaran estos aspectos como parte de las asignaturas profesionales, así la "Gestión del Cuidado" se puede analizar con una mirada disciplinar y no como actividades que se desarrollan paralelamente.

\section{CONCLUSIONES.}

De los conceptos, visiones y análisis presentados en este documento es posible concluir lo siguiente: 
1. La reforma de salud chilena, plantea modificaciones hacia la gestión de los servicios sanitarios y el sistema de atención; los fundamentos que le subyacen son mejorar la salud de la población cuantitativa y cualitativamente y reducir las desigualdades de acceso.

2. Las actividades de competencia de enfermería adquirieron en Chile el nombre jurídico de "Gestión del Cuidado" a partir de su incorporación en el código sanitario, y su importancia ha sido reconocida al establecerlo como requisito mínimo para la creación y funcionamiento de los EAR.

3. Los EAR durante el presente año, estarán en la mira de todos los actores sociales, ya que deben dar respuesta a los procesos de acreditación como forma de aseguramiento de la calidad, esta es una gran oportunidad para que las Subdirecciones de Gestión del Cuidado se destaquen liderando este proceso, y aportando desde la visión del cuidado profesional.

4. En este nuevo contexto, Enfermería debe mantener su visión desde la disciplina, utilizando la Gestión del Cuidado como el medio para mejorar los cuidados entregados.

5. La búsqueda bibliográfica no dio cuenta de evidencias para determinar si la implementación de las Subdirecciones de Gestión del Cuidado han generado algún impacto en la calidad de los cuidados otorgados a los usuarios. Se espera que los procesos de acreditación aporten experiencias publicables.

6. La mayor parte de las Carreras de Enfermería no han explicitado en sus perfiles de egreso competencias en Gestión del Cuidado, se sugiere utilizar los procesos de innovación curricular para incorporarlos.

\section{REFERENCIAS BIBLIOGRÁFICAS}

1. Artaza O. Los desafíos de la autogestión hospitalaria. Revista Chilena de Pediatría. 2008; 79 (2): 127-30.

2. Donabedian A. La dimensión internacional de la evaluación y la garantía de la calidad. Salud Pública Mex. 1990; 32 (2): 113-7.

3. Ministerio de Salud. Objetivos sanitarios para la década 2000 - 2010. El Vigía [en línea] 2002. [fecha de acceso 25 de abril 2011]; 5 (15): 3-12. Disponible en: http://epi.minsal.cl/epi/html/elvigia/vigia15.pdf

4. Vergara M, Bisama L. Autogestión hospitalaria en red I: Competencias esenciales para la gestión. Revista Chilena de Salud Pública. 2009; 13 (3): 163-8.

5. Ley № 19.937, que modifica el D.L. № 2.763, de 1979, con la finalidad de establecer una nueva concepción de la autoridad sanitaria. D.O. [en línea] 2004. [fecha de acceso 20 de abril 2011]. Disponible en: http://www.sqingenieria.cl/biblioteca/Normativa Prevencion/ley autoridadsanitaria.pdf

6. Retamal M. Autogestión de hospitalaria en cifras. Revista Chilena de Salud Pública. 2009; 13 (3): 169-74.

7. Méndez C, Torres M. Autonomía en la gestión hospitalaria en Chile: los desafíos para el recurso humano en Salud. Revista Saúde Pública [en línea] 2010. [fecha de acceso 25 de abril 2011]; 44 (2): 366-71. Disponible en: http://www.scielosp.org/pdf/rsp/v44n2/19.pdf

8. Ministerio de Salud. Decreto № 38. Reglamento orgánico de los establecimientos de salud de menor complejidad y de los establecimientos de autogestión en red. [en línea] 2005. [fecha de acceso 20 de abril 2011]. Disponible en: http://www.supersalud.gob.cl/normativa/571/ articles-4792 recurso 1.pdf

9. Vergara M, Bisama L. Autogestión hospitalaria en red II: El Método. Revista Chilena de Salud Pública. 2010; 14 (1): 52-8. 
10. Capstick R, Tarride M. Sistema de control de gestión de hospitales públicos: reflexiones para una cultura de autorregulación distribuida. Revista Chilena de Salud Pública. 2008; 12 (3): 169-80.

11. Ministerio de Salud. Decreto № 15. Reglamento del sistema de acreditación para los prestadores institucionales de salud. [en línea] 2007. [fecha de acceso 20 de abril 2011]. Disponible en: http://cursos.puc.cl/fes11/almacen/1302168839 rpobletu sec4 pos0.pdf

12. Ministerio de Salud. Estadísticas de prestadores acreditados. [en línea] 2011. [fecha de acceso 2 de mayo 2011]. Disponible en: http://www.supersalud.gob.cl/documentacion/569/ w3-article-6963.html

13. Ministerio de Salud. Compromiso de gestión 18 "Proceso de acreditación prestadores institucionales de atención cerrada de establecimientos autogestionados en red". Orientaciones Técnicas Compromisos de Gestión 2011. [en línea] 2011. [fecha de acceso 25 de abril 2011]. Disponible en: http://www.ssarauco.cl/CG2011/orientacionestec/OT CG CALIDAD/OT CALIDAD.pdf

14. Fortes $\mathrm{M}$, Araujo M, Warga T. Acreditación o la acreditación? Un estudio comparativo de la acreditación en Francia, el Reino Unido y Cataluña. Revista de la Asociación Médica Brasilera. 2011; 57 (2): 366-71.

15. Pepper S. Modelos de Acreditación. Medwave [en línea] 2011. [fecha de acceso 27 de abril 2011]; 11 (4). Disponible en: http://www.mednet.cl/link.cgi/Medwave/Series/GES03-A/4971

16. Penha E, Anselmi M, Dalmas J. Evaluación de la calidad de los procedimientos curativos en pacientes internados en un hospital universitario. Revista Latinoamericana de Enfermería. 2008; 16 (1): 57-63.

17. Ministerio de Salud. Manual del estándar general de acreditación para prestadores institucionales de atención cerrada. [en línea] 2007. [fecha de acceso 20 de abril 2011]. Disponible

en: http://200.68.46.106/doc/documentos/gestion/Acreditacion/Manual Atencion Cerrada.pdf

18. Código Sanitario. Decreto con Fuerza de Ley № 725. Actualizado a julio de 2000. Libro V, Artículo 113, inciso cuarto. [fecha de acceso 20 de abril 2011]. Disponible en:

www.bibliotecadelcongreso.cl/.../pub leyes mas soli/admin/ver archivo codigos.p hp?id codigo $=6 \&$ file $=1$

19. Subsecretaria de Redes Asistenciales, Departamento de Asesoría Jurídica. Aprueba norma general administrativa No 19, "Gestión del cuidado de Enfermería para la Atención Cerrada". [en línea] 2007. [fecha de acceso 20 de abril 2011]. Disponible

en: http://www.colegiodeenfermeras.cl/index.php?option=com k2\&view=item\&id=89:re solucion-1127-y-norma-n-19-sobre-gestión-del-cuidado-en-el-área-hospitalaria

20. Colegio de enfermeras AG. Avanza en el país la Gestión del Cuidado: El liderazgo de enfermería fortalece el compromiso con una salud pública de calidad. Enfermería 2009; Año XLIV (137):15-24.

21. Milos $P$, Bórquez B, Larraín A. La "Gestión del Cuidado" en la legislación Chilena: Interpretación y alcance. Ciencia y Enfermería. 2010; XVI (1): 17-29.

22. Estefo $S$, Paravic T. Enfermería en el rol de gestora de los cuidados. Ciencia y Enfermería. 2010; XVI (3): 33-9.

23. Milos $P$, Larraín $A$, Simonetti $M$. Categorización de servicios de enfermería, propuesta para asegurar una atención de calidad en tiempos de escasez de enfermeras. Ciencia y Enfermería. 2009; XV (1): 17-24. 
24. De Oliveira $P$, Kowal I. Perfil y competencias de gerentes de enfermería de hospitales acreditados. Revista Latinoamericana de Enfermería. 2011; 19 (1): 10614.

25. U.S Department of Health \& Human Services. Agency for Helthcare Research and Quality. Los cuidados supervisados por enfermeras mejoran el funcionamiento vital de pacientes con insuficiencia cardiaca que residen en comunidades minoritarias. [en línea] 2006. [fecha de acceso 2 de mayo 2011]. Disponible en: http://www.ahrq.gov/news/press/pr2006/spnursepr. htm

26. Agramonte A, Farres R. Influencia de la gestión del cuidado en la calidad de la atención en salud. Revista Cubana de Enfermería. 2010; 26 (2): 14-26.

27. Torres E, Lastra J. Propuesta de una escala para medir la calidad del servicio de los centros de atención secundaria de salud. Revista de Administração Pública. 2008; 42 (4): 719-34.

28. Bautista L. Percepción de la calidad del cuidado de enfermería en la ESE Francisco de Paula Santander. Aquichan. 2008; 8 (1): 74-84.

29. Sierra C, Muñoz A, Peiró M, Valls S, López I, Famoso M. Elaboración de un cuestionario para medir la calidad con los cuidados de enfermería en unidades de cuidados intensivos cardiológicos desde la percepción de los pacientes. Enfermería en Cardiología. 2009; XVI (47-48): 71-9.

30. Velásquez R, Flores J, Picallos M, Acosta M. Calidad asistencial percibida por los padres en una unidad de preescolares y hemato-oncología pediátrica. Index de Enfermería. 2010; 19 (1): 34-36.

31. Ponce J, Reyes H, Ponce G. Satisfacción laboral y calidad de atención de enfermería en una unidad médica de alta complejidad. Revista de Enfermería del Instituto Mexicano del Seguro Social. 2006; 14 (2): 65-73.

32. Mendes $\mathrm{G}$. La dimensión ética de la acción y las cuestiones relativas a la calidad de los cuidados de enfermería. Texto y Contexto. 2009; 18 (1): 165-9.

33. Ceballos $P$. Desde los ámbitos de Enfermería, analizando el cuidado humanizado. Ciencia y Enfermería. 2010; XVI (1): 31-5.

34. Watson J. Ponencia del primer congreso internacional de Enfermería. Facultad de Enfermería y Nutriología. Universidad Autónoma de Chihuahua. [fecha de acceso 02 de mayo 2011]. Disponible en: http://fen.uach.mx/index.php?page/Semblanza Jean Watson 